Article

\title{
A New Flexible Method for Solving Multi-Expert Multi-Criterion Decision-Making Problems
}

\author{
Ta-Chun Wen ${ }^{1}(0)$ Hsin-Hung Lai ${ }^{2}\left(\mathbb{D}\right.$ and Kuei-Hu Chang ${ }^{3,4, *}$ \\ 1 Yango University, Fuzhou 350015, China; dj.wen@yahoo.com.tw \\ 2 Department of Civil Engineering, R.O.C. Military Academy, Kaohsiung 830, Taiwan; \\ kevin5485xd@gmail.com \\ 3 Department of Management Sciences, R.O.C. Military Academy, Kaohsiung 830, Taiwan \\ 4 Institute of Innovation and Circular Economy, Asia University, Taichung 413, Taiwan \\ * Correspondence: evenken2002@yahoo.com.tw
}

Received: 12 June 2020; Accepted: 29 June 2020; Published: 1 July 2020

\begin{abstract}
Multi-expert multi-criterion decision-making problems are complicated decision issues. Different experts have different opinions with regard to multi-criterion decision-making problems, depending on their background and experience. These experts provide information that usually contains certain, uncertain, and incomplete information simultaneously. However, traditional computing methods only fully consider certain information, and ignore uncertain or incomplete information, which causes information distortion in assessment results. In order to effectively solve the above problems, this paper proposes a new flexible method for solving multi-expert multi-criterion decision-making problem. The primary purpose of the proposed method is to fully consider all information provided by experts to avoid information distortion of assessment results. Finally, an illustrative example of computer numerical control (CNC) machine tool selection is provided to prove the practicality of the proposed method. After comparing the results generated by proposed method with the results generated using linguistic ordered weighted geometric averaging operator (LOWGA) operator and induced LOWGA operator methods, the results indicate that the proposed method integrating an induced LOWGA operator and a hesitant fuzzy linguistic term set is more flexible and is able to reflect real-world situations.
\end{abstract}

Keywords: computer numerical control; induced linguistic ordered weighted geometric averaging operator; hesitant fuzzy linguistic term set; group decision making

\section{Introduction}

Multi-expert multi-criterion decision-making (MCDM) problems are complex decision-making (DM) problems with both qualitative and quantitative characteristics. In recent years, many researchers have examined the related issues of multi-expert MCDM [1-12]. For instance, Liu et al. [13] proposed the generalized ordered hybrid modular operator based on a generalized ordered modular averaging operator to handle multi-attribute group DM problems. Here, the operator considers not only the importance level of the input parameter, but also its sequence position. For handling group DM problems, an induced-language continuous ordered weighted geometry (OWG) operator with uncertain multiplicative language preference relations was proposed by Zhou and Chen [14]. Gitinavard et al. [15] used an interval-valued hesitant fuzzy set to propose a new multi-criterion weighting ranking method to solve complex group DM issues. Ghorabaee et al. [16] extended the distance from the average solution method to handle issues of supplier selection in fuzzy environments.

Group DM is about situations in which multiple individuals act collectively to achieve a solution in managerial practice. Because of the uncertainty and complexity of group DM problems, 
expert-provided information usually contains uncertain information. To effectively resolve this issue, Rodriguez et al. [17] introduced the hesitant fuzzy linguistic term set (HFLTS) to handle situations where experts are hesitating between several possible linguistic term sets for evaluating a variable, indicator, alternative, etc. The HFLTS method is a derivative of hesitant fuzzy sets [18]. Since it appeared, many researchers have developed applications and extensions of HFLTS in different fields. For example, Beg and Rashid [19] used HFLTS and applied the technique to order preference by similarity to ideal solution (TOPSIS) to solve an investment company selection problem and allow the company to invest money in the optimal selection. Based on HFLTS, Wang et al. [20] introduced an outranking method to survey issues of offensive warfare capabilities in information warfare. Offensive warfare capabilities include information suppression, hard destruction, network resistance, and psychological resistance. Montes et al. [21] applied HFLTS to propose a linguistic multi-expert MCDM model for housing markets. Ashtiani and Azgomi [22] considered the uncertainty, vagueness, and hesitancy of the provided opinions to propose a computational trust model based on HFLTS. Based on HFLTS, TOPSIS, and analytic hierarchy process methods, Onar et al. [23] introduced a new fuzzy quality function deployment method to effectively determine the best computer workstation. To date, HFLTS has received lots of attention and has been applied in many applications [24-29].

Ordered weight is an important factor in multi-expert MCDM problems and preference ranking [30]. The concept of ordered weight was first introduced by Yager [31], who used an ordered weighted averaging (OWA) operator to solve multi-expert MCDM problems. An OWA operator is a critical aggregation operator that generates an aggregating result ranging from minimum to maximum. The ordered weighted geometric averaging (OWGA) operator uses the geometric mean OWA operator concept to generate an aggregating result for the information. Recently, OWA operators and OWGA operators have been discussed in different fields and extensions [32-39].

Many studies have used different calculation methods to handle group DM issues [2,5-9,11,12]. However, these methods cannot handle uncertain or incomplete information being included in the aggregated information. The process of information aggregation may involve partially missing data or unobtainable information. If only the complete information is considered, sample numbers will be reduced, and information distortion will occur. To effectively resolve this issue as it related to group $\mathrm{DM}$, this study proposes a more general approach: the integration of an induced linguistic ordered weighted geometric averaging (LOWGA) operator and HFLTS. To demonstrate the practicality of the proposed method, an illustrative example of computer numerical control (CNC) machine selection tool is presented in this paper. The calculation result achieved by the proposed approach was also compared with those achieved by LOWGA operator and induced LOWGA operator approaches.

The rest of the article is organized as follows. Section 2 outlines some fundamental concepts related to OWA, OWGA, LOWGA, and induced LOWGA operators, and HFLTS is briefly reviewed. Section 3 proposes a novel method that integrates an induced LOWGA operator and HFLTS. An illustrative example validating the proposed method is be discussed in Section 4. In Section 5, conclusions are given.

\section{Preliminaries}

\subsection{OWA, OWGA, LOWGA, and Induced LOWGA Operators}

The basic concept of OWA, OWGA, LOWGA, and induced LOWGA operators is the re-ordering step. Yager [31] first proposed the OWA operator concept to handle MCDM problems, which can be explained as follows. 
Definition 1 [40]. The OWA operator is a mapping $O W A: R^{n} \rightarrow R$ with dimension $n$, which is associated with a weighting vector $w=\left(w_{1}, w_{2}, \ldots, w_{n}\right)^{T}$ and $\sum_{j=1}^{n} w_{j}=1$, and has the properties

$$
\mathrm{OWA}\left(a_{1}, a_{2}, \ldots, a_{n}\right)=\sum_{j=1}^{n} w_{j} b_{j}
$$

where $b_{j}$ is the jth largest element in the set $\left\{a_{1}, a_{2}, \ldots, a_{n}\right\}$.

The OWGA operator is based on the OWA operator and geometric mean.

Definition 2 [41]. The OWGA operator is a mapping OWGA : $R^{+n} \rightarrow R^{+}$with dimension $n$, which is associated with a weighting vector $w=\left(w_{1}, w_{2}, \ldots, w_{n}\right)^{T}$ and $\sum_{j=1}^{n} w_{j}=1$, and has the properties

$$
\operatorname{OWGA}\left(a_{1}, a_{2}, \ldots, a_{n}\right)=\prod_{j=1}^{n} b_{j}^{w_{j}}
$$

where $b_{j}$ is the jth largest element in the $\left\{a_{1}, a_{2}, \ldots, a_{n}\right\}$ set.

For managing linguistic information, $\mathrm{Xu}$ [42] proposed the LOWGA operator to extend the concept of the OWGA operator to a linguistic environment. It can be explained as follows.

Definition 3 [43]. The LOWGA operator is a mapping LOWGA: $\bar{S}^{n} \rightarrow \bar{S}$ with dimension $n$, which is associated with a weighting vector $w=\left(w_{1}, w_{2}, \ldots, w_{n}\right)^{T}$ and $\sum_{j=1}^{n} w_{j}=1$, and which has the properties:

$$
\begin{gathered}
\operatorname{LOWG} A_{w}\left(s_{\alpha_{1}}, s_{\alpha_{2}}, \ldots, s_{\alpha_{n}}\right)=\left(s_{\beta_{1}}\right)^{w_{1}} \otimes\left(s_{\beta_{2}}\right)^{w_{2}} \otimes \ldots \otimes\left(s_{\beta_{n}}\right)^{w_{n}} \\
=\left(s_{\beta_{1} w_{1}}\right) \otimes\left(s_{\beta_{2} w_{2}}\right) \otimes \ldots\left(s_{\beta_{n} w_{n}}\right)=s_{\beta}
\end{gathered}
$$

where $\otimes$ expressed the product symbol for numbers, $\beta=\prod_{j=1}^{n} b_{j}^{w_{j}}$, and $s_{\beta_{j}}$ is the jth largest element in the set $\left\{s_{\alpha_{1}}, s_{\alpha_{2}}, \ldots, s_{\alpha_{n}}\right\}$

Extending the concept of the LOWGA operator, Wei et al. [43] proposed the induced LOWGA operator (ILOWGA), and considered both the weights related to the weight of the attributes and that of the operators synchronously.

Definition 4 [44]. An induced LOWGA operator can be explained as follows.

$$
\operatorname{ILOWG} A_{\bar{w}, w}\left(s_{\alpha_{1}}, s_{\alpha_{2}}, \ldots, s_{\alpha_{n}}\right)=\left(s_{\beta_{1}}\right)^{w_{1}} \otimes\left(s_{\beta_{2}}\right)^{w_{2}} \otimes \ldots \otimes\left(s_{\beta_{n}}\right)^{w_{n}}=s_{\beta}
$$

where $\bar{w}=\left(\bar{w}_{1}, \bar{w}_{2}, \ldots, \bar{w}_{n}\right)^{T}$ is the weighting of the $s_{\alpha}$ satisfying $\sum_{j=1}^{n} \bar{w}_{j}=1$ and $\bar{w}_{j} \in[0,1]$.

$w=\left(w_{1}, w_{2}, \ldots, w_{n}\right)^{T}$ is the exponential weighting vector of the $s_{\beta}$ satisfying $\sum_{j=1}^{n} w_{j}=1$ and $w_{j} \in[0,1] . \beta=\prod_{j=1}^{n} b_{j}^{w_{j}}, s_{\beta_{j}}$ is the $j$ th largest element in the $\left\{\bar{s}_{\alpha_{1}}, \bar{s}_{\alpha_{2}}, \ldots, \bar{s}_{\alpha_{n}}\right\}$ set, i.e.,

$$
\bar{s}_{\alpha_{i}}=r \bar{w}_{i} s_{\alpha_{i}}=s_{r \bar{w}_{i}} s_{\alpha_{i}}
$$

in which $i=1,2, \ldots, n, r$ is the balancing coefficient, and the value of $r$ is usually equal to $n$. 


\subsection{Hesitant Fuzzy Linguistic Term Set}

Extending the concept of fuzzy sets, Torra [18] proposed the hesitant fuzzy set (HFT) in order to manage situations containing hesitation in determining the membership degree of the elements in human decision-making processes. HFT can be explained as follows.

Definition 5 [18]. If $X$ is a reference set, regarding the HFT $E$ of $X$, this function returns a subset of $[0,1]$ when applied to $X$, can be defined as follows.

$$
E=\left\{\left\langle x, h_{E}(x)\right\rangle \mid x \in X\right\}
$$

where $h_{E}(x)$ represents the degree of membership of elements $x \in X$ to set $E$, and its values are in the interval $[0,1]$.

Rodriguez et al. [17] first introduced HFLTS to optimize the computational capabilities of conventional linguistic methods, in situations wherein experts hesitate between some values for evaluating data [45,46]. The basic concept of the HFLTS is based on the HFT and fuzzy linguistic approaches, and can be explained as follows.

Definition 6 [21]. An HFLTS $\left(H_{S}\right)$ is an ordered finite subset of continuous semantic terms of $S$, $S=\left\{s_{0}, s_{1}, \ldots, s_{g}\right\}$.

Definition 7 [21]. The full HFLTS and empty HFLTS for a semantic variable $\alpha$ are explained as follows.

$$
\text { Full HFLTS : } H_{s}(\alpha)=S \text {; }
$$$$
\text { Empty HFLTS : } H_{s}(\alpha)=\varnothing \text {. }
$$

Definition 8 [47]. Let $S=\left\{s_{0}, s_{1}, \ldots, s_{g}\right\}$ be a linguistic term set. $H_{S}, H_{S^{\prime}}^{1}$ and $H_{S}^{2}$ are the arbitrary HFLTS on $S$, and then their intersection, union, and complement operations are explained as follows.

$$
\begin{gathered}
H_{S}^{1} \cap H_{S}^{2}=\left\{s_{i} \mid s_{i} \in H_{S}^{1} \text { and } s_{i} \in H_{S}^{2}\right\} ; \\
H_{S}^{1} \cup H_{S}^{2}=\left\{s_{i} \mid s_{i} \in H_{S}^{1} \text { or } s_{i} \in H_{S}^{2}\right\} ; \\
H_{S}^{C}=S-H_{S}=\left\{s_{i} \mid s_{i} \in S \text { and } s_{i} \notin H_{S}\right\} .
\end{gathered}
$$

Definition 9 [17]. The upper bound $H_{s^{+}}$and lower bound $H_{s^{-}}$of HFLTS are explained as follows.

$$
\begin{aligned}
& H_{s^{+}}=\max \left(s_{i}\right)=s_{j}, s_{i} \in H_{s}, \text { and } s_{i} \leq s_{j} \forall i ; \\
& H_{s^{-}}=\min \left(s_{i}\right)=s_{j}, s_{i} \in H_{s}, \text { and } s_{i} \geq s_{j} \forall i .
\end{aligned}
$$

Definition 10 [47]. The envelope of an HFLTS, env $\left(H_{S}\right)$, is a linguistic interval, which is restricted by its lower bound $\mathrm{H}_{\mathrm{s}^{-}}$and upper bound $\mathrm{H}_{\mathrm{s}^{+}}$.

$$
\operatorname{env}\left(H_{s}\right)=\left[H_{s^{-}}, H_{S^{+}}\right]
$$

\section{Proposed Integration of Induced LOWGA Operator and HFLTS}

Multi-expert MCDM problems are complex, and include uncertainty in group DM. In real decision-making, the criterion weights and criterion rating values for alternatives usually simultaneously contain certain, uncertain, or incomplete information. This is because the experts' opinions include personal preferences according to their different backgrounds and experience. 
Traditional linguistic computing methods may cause information distortion and loss in the information aggregation process. To address this problem, this paper presents the integration of an induced LOWGA operator and HFLTS to augment the assessment ability in group DM problems. In contrast to other methods, the proposed method can effectively deal with the criterion weights and criterion rating values for alternative or uncertain and incomplete information. For uncertain information provided by experts, the HFLTS approach is used to increase the flexibility of linguistic expression in assessments. For incomplete information provided by experts, existing complete information is used with supplementing information to fill gaps in incomplete information.

The process of the proposed novel group DM method is divided into the following seven steps (shown in Figure 1).

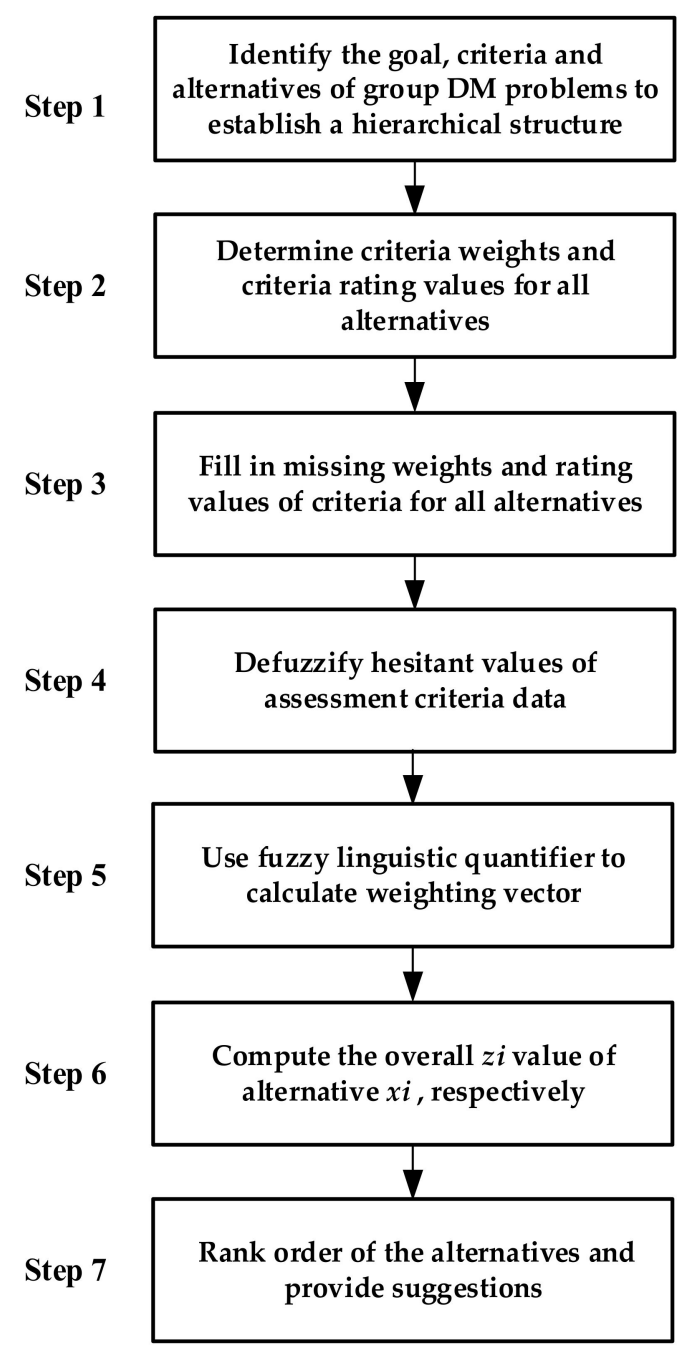

Figure 1. The flow chart of proposed novel group decision-making (DM) method.

Step 1. Identify the goal, criteria, and alternatives of group DM problems to establish a hierarchical structure.

According to the problems to be evaluated, the decision-making committee must disassemble problems into hierarchical structures that include alternatives, criteria, and goals.

Step 2. Determine criterion weights and criterion rating values for all alternatives.

According to decision-making committee members' different knowledge and experience, each committee member individually provides different assessment weights and rating values of criteria for all alternatives. 
Step 3. Fill in missing weights and rating values of criteria for all alternatives.

If the information provided by experts is incomplete, incomplete information can be supplemented by the mean value of all existing complete information. If all information is complete, move to Step 4 .

Step 4. Defuzzify hesitant values of assessment criterion data.

In this step, the parametric mean (PM) is used to defuzzify the hesitant values of assessment criterion data [45], computed as shown in Equation (6).

$$
\mathrm{PM}=\left(\frac{a+b}{2}\right)
$$

where $a$ and $b$ represent left boundaries and right boundaries, respectively.

Step 5. Use fuzzy linguistic quantifier to calculate weighting vector.

The aggregation weighting vector $w=\left(w_{1}, w_{2}, \ldots, w_{n}\right)^{T}$ of the proposed method using fuzzy quantifiers, which for a non-decreasing relative quantifier $Q$, is defined as [48,49]

$$
\begin{gathered}
w_{j}=Q\left(\frac{j}{n}\right)-Q\left(\frac{j-1}{n}\right), j=1,2, \ldots, n \\
(r)=\left\{\begin{array}{cc}
0 & \text { if } r<a \\
\frac{r-a}{b-a} & \text { if } a \leq r \leq b \quad \text { if } r<b \\
1 & \text { if } r b, r \in[0,1]
\end{array}\right.
\end{gathered}
$$

Three frequently used fuzzy quantifiers "at least", "most", and "as many as possible" are utilized to obtain the weighting vector $w=\left(w_{1}, w_{2}, \ldots, w_{n}\right)^{T}$, of which the parameters $(a, b)$ are $(0,0.5),(0.3,0.8)$ and $(0.5,1)$, respectively.

Step 6. Compute the overall $z_{i}$ value of alternative $x_{i}$, respectively [43].

$$
z_{i}=I L O W G A_{\bar{w}, w}\left(s_{\alpha_{i 1}}, s_{\alpha_{i 2}}, \ldots, s_{\alpha_{i n}}\right)
$$

Using the results of Steps 3 to 5, use Equation (9) to calculate overall value $z_{i}$ of alternative $A_{i}$.

Step 7. Rank order of the alternatives and provide suggestions.

According to the calculation results of Step 6, rank the order of the alternatives and provide feasible suggestions for decision-makers.

\section{Numerical Verification and Comparison}

\subsection{Overview}

An illustrative example of CNC machine tool selection (adapted from Reference [50]) was used in this section to demonstrate the merit of the proposed method. This study also compared results generated using the LOWGA operator and ILOWGA operator methods. CNC is a computer-aided design protocol that uses computers to control machine tools. Selecting the most appropriate CNC machine tool for the Pakistan Machine Tool Factory Pte Ltd can improve product reliability and increase overall productivity [50]. This company started production in 1971, and company management hoped to choose the optimal CNC machine tool to pursue the largest output with the least cost. The hierarchical structure for selecting the optimal CNC machine tool is shown in Figure 2. As shown in Figure 2, the $\mathrm{CNC}$ machine tool selection included six criteria and five alternatives. The six criteria used to evaluate the alternatives were capital cost (C1), spindle speed (C2), bar capacity (C3), horsepower (C4), agility (C5), and turning meter (C6). The five alternatives were Mazak (A1), Romi (A2), Dossan (A3), Nakamura (A4), and Saim (A5). 


\section{Select the suitable computer numerical control machine tool}

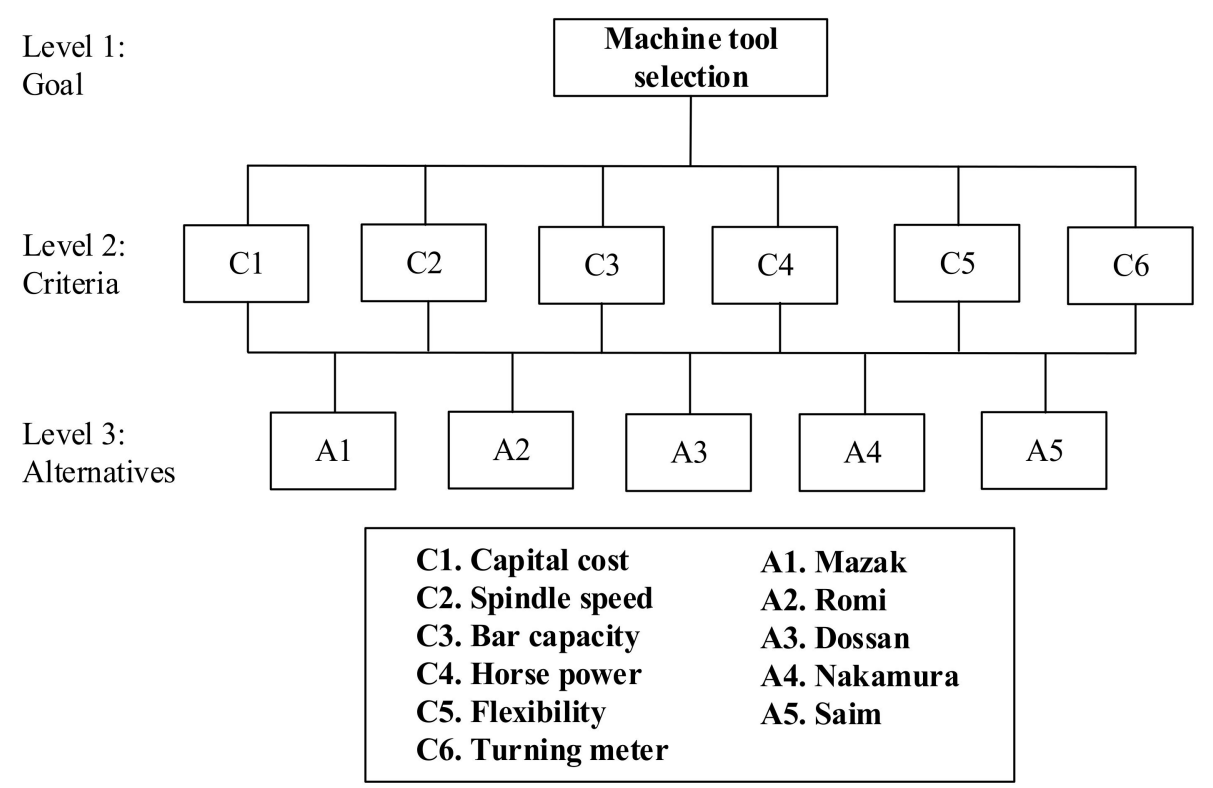

Figure 2. Hierarchical structure for selecting the optimal computer numerical control (CNC) machine tool.

In this case, the decision-making committee for selecting the optimal CNC machine tool included four experts $D k(k=1,2, \ldots, 4)$, chosen based on their backgrounds and experience, each providing different assessment criterion weights and criterion rating values for the five alternatives, as shown in Tables 1 and 2. The four experts include a manager, one outside expert, and two engineers from the factory.

Appropriate semantic variables of the criterion weights scale were as follows.

$$
\left.L_{1}=\left\{s_{1}: V U(\text { very unimportant }), s_{2}: U(\text { unimportant }), s_{3}: M(\text { medium }), s_{4}: I(\text { important }), s_{5}: \text { VI(very important }\right)\right\} .
$$

The appropriate semantic variables regarding the scales of the criterion rating values of the five alternatives were as follows.

$$
\begin{gathered}
L_{2}=\left\{s_{1}: V B(\text { very bad }), s_{2}: B(\text { bad }), s_{3}: M B(\text { medium bad }), s_{4}: M(\text { medium })\right. \\
\left.s_{5}: M G(\text { medium good }), s_{6}: G(\text { good }), s_{7}: G V(\text { very good })\right\} .
\end{gathered}
$$

Table 1. Semantic variables of the criterion weights for the five alternatives.

\begin{tabular}{ccccccc}
\hline & $C 1$ & $C 2$ & $C 3$ & $C 4$ & $C 5$ & $C 6$ \\
\hline$D 1$ & VI & I & M & VI & M & VI \\
$D 2$ & VI & M & $*$ & VI & $*$ & {$[\mathrm{I}, \mathrm{VI}]$} \\
$D 3$ & VI & I & VI & M & I & I \\
$D 4$ & {$[\mathrm{I}, \mathrm{VI}]$} & $\mathrm{M}$ & $\mathrm{VI}$ & {$[\mathrm{I}, \mathrm{VI}]$} & {$[\mathrm{M}, \mathrm{I}]$} & $\mathrm{I}$ \\
\hline \multicolumn{7}{c}{ The missing values are shown with } \\
\end{tabular}

The missing values are shown with *. 
Table 2. Criterion rating values for five alternatives.

\begin{tabular}{|c|c|c|c|c|c|c|c|}
\hline \multirow{2}{*}{ Decision-Makers } & \multirow{2}{*}{ Alternatives } & \multicolumn{6}{|c|}{ Criterion } \\
\hline & & $C 1$ & $C 2$ & $C 3$ & $C 4$ & $C 5$ & C6 \\
\hline \multirow{5}{*}{$D 1$} & $A 1$ & M & VG & $M$ & B & $\mathrm{MB}$ & MG \\
\hline & $A 2$ & G & VG & MB & VG & MG & G \\
\hline & $A 3$ & VG & $\mathrm{MB}$ & G & $\mathrm{M}$ & MB & G \\
\hline & $A 4$ & $\mathrm{G}$ & $\mathrm{M}$ & MG & MG & G & G \\
\hline & $A 5$ & VG & G & VG & MG & MG & G \\
\hline \multirow{5}{*}{$D 2$} & $A 1$ & G & $M$ & * & $M$ & * & G \\
\hline & $A 2$ & G & G & * & MG & MG & {$[G, V G]$} \\
\hline & $A 3$ & VG & $\mathrm{MB}$ & MG & $\mathrm{M}$ & $\mathrm{M}$ & $G$ \\
\hline & $A 4$ & $\mathrm{G}$ & $\mathrm{MB}$ & MG & G & MG & {$[\mathrm{MG}, \mathrm{G}]$} \\
\hline & $A 5$ & G & VG & G & VG & * & {$[\mathrm{MG}, \mathrm{G}]$} \\
\hline \multirow{5}{*}{ D3 } & $A 1$ & MG & VG & MG & $M$ & M & G \\
\hline & $A 2$ & VG & G & MB & G & MG & G \\
\hline & $A 3$ & VG & MG & VG & MB & $\mathrm{M}$ & G \\
\hline & $A 4$ & VG & $\mathrm{M}$ & MG & MG & M & MG \\
\hline & $A 5$ & G & VG & VG & MG & M & G \\
\hline \multirow{5}{*}{$D 4$} & $A 1$ & {$[\mathrm{MB}, \mathrm{M}]$} & $G$ & $\mathrm{M}$ & $\mathrm{M}$ & $\mathrm{MB}$ & G \\
\hline & $A 2$ & G & VG & M & G & G & MG \\
\hline & $A 3$ & VG & $\mathrm{MB}$ & MG & {$[\mathrm{MB}, \mathrm{M}]$} & M & MG \\
\hline & $A 4$ & G & $\mathrm{M}$ & MG & $\mathrm{G}$ & MG & VG \\
\hline & $A 5$ & {$[\mathrm{G}, \mathrm{VG}]$} & G & VG & G & {$[\mathrm{M}, \mathrm{MG}]$} & G \\
\hline
\end{tabular}

The missing values are shown with *.

\subsection{Solution from the LOWGA Operator Method}

When input arguments are semantic variables, to deal with the semantic information, $\mathrm{Xu}$ [42] proposed the LOWGA operator. The LOWGA operator is an extension of OWGA operator used to aggregate semantic information. The assessment of criterion weights and criterion rating values for expert-provided information requires certain information in the LOWGA operator method. In this case, some information included uncertain or incomplete information from experts D2 and D4. Therefore, this method considered only the complete information of experts D1 and D3. For example, the criterion rating values for alternative $A_{1}$ were $4.5000,7.000,4.5000,3.000,3.5000$, and 5.500, respectively. Sorting from largest to smallest gave 7.000, 5.500, 4.5000, 4.5000, 3.5000, and 3.000.

Based on the LOWGA operator method, using Equation (3), Table 1, and Table 2, we calculated the overall $z_{i}$ value of alternative $A_{i}$.

$$
\begin{gathered}
z_{1}=\operatorname{LOWGA}_{w}\left(s_{4.5}, s_{7}, s_{4.5}, s_{3}, s_{3.5}, s_{5.5}\right) \\
=\left(s_{7.000}\right)^{0} \otimes\left(s_{5.500}\right)^{0.067} \otimes\left(s_{4.500}\right)^{0.333} \otimes\left(s_{4.500}\right)^{0.333} \otimes\left(s_{3.500}\right)^{0.267} \otimes\left(s_{3.000}\right)^{0} \\
=s_{\left(7.000^{0} \times 5.500^{0.067} \times 4.500^{0.333} \times 4.500^{0.333} \times 3.500^{0.267} \times 3.000^{0}\right)} \\
=s_{4.265}
\end{gathered}
$$

The other values were obtained through the LOWGA operator method.

$z_{2}=s_{5.901}, z_{3}=s_{4.564}, z_{4}=s_{5.000}$, and $z_{5}=s_{5.901}$.

According to the LOWGA operator method, the ranking order of the alternative was

$$
A_{5}=A_{2}>A_{4}>A_{3}>A_{1}
$$




\subsection{Solution from the Induced LOWGA Operator Method [43]}

Based on the LOWGA operator, Wei et al. [43] proposed a more general model for an aggregation operator, the induced LOWGA operator, which considers the weights associated with both operators and attributes. As in the LOWGA operator method, the induced LOWGA operator method requires that assessment information be certain information. Because the partial information provided by experts D2 and D4 was uncertain or incomplete, we considered only the complete information of experts D1 and D3.

According to Equations (7) and (8), we used the fuzzy linguistic quantifier "Most" to get the weighting vector, as shown below.

$$
\begin{gathered}
w_{j}=Q\left(\frac{j}{n}\right)-Q\left(\frac{j-1}{n}\right) \\
w_{1}=Q\left(\frac{1}{6}\right)-Q\left(\frac{1-1}{6}\right)=0-0=0 \\
w_{2}=Q\left(\frac{2}{6}\right)-Q\left(\frac{2-1}{6}\right)=0.067-0=0.067 \\
w_{3}=Q\left(\frac{3}{6}\right)-Q\left(\frac{3-1}{6}\right)=0.4-0.067=0.333 \\
w_{4}=Q\left(\frac{4}{6}\right)-Q\left(\frac{4-1}{6}\right)=0.733-0.4=0.333 \\
w_{5}=Q\left(\frac{5}{6}\right)-Q\left(\frac{5-1}{6}\right)=1-0.733=0.267 \\
w_{6}=Q\left(\frac{6}{6}\right)-Q\left(\frac{6-1}{6}\right)=1-1=0
\end{gathered}
$$

Wei et al. [43] utilized the $z_{i}$ value to identify the ranking order of alternative tools. Based on Table 1, Table 2, Equation (4), and Equation (5), we calculated the $z_{i}$ values.

$$
\begin{gathered}
z_{1}=I L O W G A_{\bar{w}, w}\left(s_{\alpha_{11}}, s_{\alpha_{12}}, \ldots, s_{\alpha_{1 n}}\right)=\operatorname{ILOWGA} A_{\bar{w}, w}\left(s_{4.5}, s_{7}, s_{4.5}, s_{3}, s_{3.5}, s_{5.5}\right) \\
=\left(s_{\beta_{1}}\right)^{0} \otimes\left(s_{\beta_{2}}\right)^{0.067} \otimes\left(s_{\beta_{3}}\right)^{0.333} \otimes\left(s_{\beta_{4}}\right)^{0.333} \otimes\left(s_{\beta_{5}}\right)^{0.267} \otimes\left(s_{\beta_{6}}\right)^{0} \\
=\left(s_{6.720}\right)^{0} \otimes\left(s_{5.940}\right)^{0.067} \otimes\left(s_{5.400}\right)^{0.333} \otimes\left(s_{4.320}\right)^{0.333} \otimes\left(s_{2.940}\right)^{0.267} \otimes\left(s_{2.880}\right)^{0} \\
=s_{\left(6.720^{0} \times 5.940^{0.067} \times 5.400^{0.333} \times 4.320^{0.333} \times 2.940^{0.267} \times 2.880^{0}\right)} \\
=s_{4.289}
\end{gathered}
$$

The other values were also obtained using the induced LOWGA operator method.

$z_{2}=s_{5.628}, z_{3}=s_{4.511}, z_{4}=s_{4.698}$ and $z_{5}=s_{5.921}$.

According to induced LOWGA operator method operation, the ranking of alternatives was $A_{5}>A_{2}>A_{4}>A_{3}>A_{1}$.

\subsection{Solution from the Proposed New Group DM Approach}

The proposed new group DM approach integrates an induced LOWGA operator and a HFLTS to increase the assessment ability of group decision-making problems. According to the judgments of committee members, a hierarchical structure of CNC machine tool selection was established. The criterion weights and criterion rating values for all alternatives were then identified, as shown in Figure 2 and Tables 1 and 2 (Steps 1 and 2).

The remaining steps of the proposed new group DM approach can be explained as follows.

Step 3. Fill in missing values of criterion weights and criterion rating values for all alternatives 
In this step, all incomplete information is supplemented by averaging all existing complete information. This results in complete criterion weights and criterion rating values for the five alternatives, as shown in Tables 3 and 4.

Table 3. Complete information of criterion weights.

\begin{tabular}{ccccccc}
\hline & C1 & C2 & C3 & C4 & C5 & C6 \\
\hline$D 1$ & 1.000 & 0.800 & 0.600 & 1.000 & 0.600 & 1.000 \\
$D 2$ & 1.000 & 0.600 & 0.867 & 1.000 & 0.700 & {$[0.8,1]$} \\
$D 3$ & 1.000 & 0.800 & 1.000 & 0.600 & 0.800 & 0.800 \\
$D 4$ & {$[0.8,1]$} & 0.600 & 1.000 & {$[0.8,1]$} & {$[0.6,0.8]$} & 0.800 \\
\hline
\end{tabular}

Table 4. Complete information of criterion rating values for alternatives.

\begin{tabular}{|c|c|c|c|c|c|c|c|}
\hline \multirow{2}{*}{$\begin{array}{l}\text { Decision- } \\
\text { Makers }\end{array}$} & \multirow{2}{*}{ Alternatives } & \multicolumn{6}{|c|}{ Criteria } \\
\hline & & $C 1$ & $C 2$ & $C 3$ & $C 4$ & $C 5$ & C6 \\
\hline \multirow{5}{*}{$D 1$} & $A 1$ & 0.571 & 1 & 0.571 & 0.286 & 0.429 & 0.714 \\
\hline & $A 2$ & 0.857 & 1 & 0.429 & 1 & 0.714 & 0.857 \\
\hline & $A 3$ & 1 & 0.429 & 0.857 & 0.571 & 0.429 & 0.857 \\
\hline & $A 4$ & 0.857 & 0.571 & 0.714 & 0.714 & 0.857 & 0.857 \\
\hline & $A 5$ & 1 & 0.857 & 1 & 0.714 & 0.714 & 0.857 \\
\hline \multirow{5}{*}{$D 2$} & $A 1$ & 0.857 & 0.571 & 0.762 & 0.571 & 0.667 & 0.857 \\
\hline & $A 2$ & 0.857 & 0.857 & 0.762 & 0.714 & 0.714 & {$[0.857,1]$} \\
\hline & $A 3$ & 1 & 0.429 & 0.714 & 0.571 & 0.571 & 0.857 \\
\hline & $A 4$ & 0.857 & 0.429 & 0.714 & 0.857 & 0.714 & $\begin{array}{c}{[0.714,} \\
0.857]\end{array}$ \\
\hline & $A 5$ & 0.857 & 1 & 0.857 & 1 & 0.667 & $\begin{array}{l}{[0.714,} \\
0.857]\end{array}$ \\
\hline \multirow{5}{*}{ D3 } & $A 1$ & 0.714 & 1 & 0.714 & 0.571 & 0.571 & 0.857 \\
\hline & $A 2$ & 1 & 0.857 & 0.429 & 0.857 & 0.714 & 0.857 \\
\hline & $A 3$ & 1 & 0.714 & 1 & 0.429 & 0.571 & 0.857 \\
\hline & $A 4$ & 1 & 0.571 & 0.714 & 0.714 & 0.571 & 0.714 \\
\hline & $A 5$ & 0.857 & 1 & 1 & 0.714 & 0.571 & 0.857 \\
\hline \multirow{5}{*}{$D 4$} & $A 1$ & {$[0.429,0.571]$} & 0.857 & 0.571 & 0.571 & 0.429 & 0.857 \\
\hline & $A 2$ & 0.857 & 1 & 0.571 & 0.857 & 1 & 0.714 \\
\hline & $A 3$ & 1 & 0.429 & 0.714 & {$[0.429,0.571]$} & 0.571 & 0.714 \\
\hline & $A 4$ & 0.857 & 0.571 & 0.714 & 0.857 & 0.714 & 1 \\
\hline & $A 5$ & {$[0.857,1]$} & 0.857 & 1 & 0.857 & {$[0.571,0.714]$} & 0.857 \\
\hline
\end{tabular}

Step 4. Defuzzify the hesitant values of assessment criterion data. Equation (6) was used to obtain the defuzzified results shown in Tables 3 and 4.

Step 5. Use the fuzzy linguistic quantifier to calculate weighting vectors.

According to Equations (7) and (8), we used the fuzzy linguistic quantifier "Most" to obtain weighting vectors.

Step 6. Calculate the overall $z_{i}$ values of alternatives $x_{i}$, respectively [43].

According to Table 4, we defuzzified the hesitant values of the assessment criterion data for CNC machine tool selection, then aggregated the expert provided information, as shown in Table 5.

Table 5. Aggregated information of criterion rating values for five alternatives.

\begin{tabular}{ccccccc}
\hline \multirow{2}{*}{ Alternatives } & \multicolumn{7}{c}{ Criterion } \\
\cline { 2 - 7 } & $\boldsymbol{C 1}$ & $\boldsymbol{C 2}$ & $\boldsymbol{C 3}$ & $\boldsymbol{C 4}$ & $\boldsymbol{C 5}$ & $\boldsymbol{C 6}$ \\
\hline$A 1$ & 0.661 & 0.857 & 0.655 & 0.500 & 0.524 & 0.821 \\
$A 2$ & 0.893 & 0.929 & 0.548 & 0.857 & 0.786 & 0.839 \\
$A 3$ & 1.000 & 0.500 & 0.821 & 0.518 & 0.536 & 0.821 \\
$A 4$ & 0.893 & 0.536 & 0.714 & 0.786 & 0.714 & 0.839 \\
$A 5$ & 0.911 & 0.929 & 0.964 & 0.821 & 0.649 & 0.839 \\
\hline
\end{tabular}


According to Table 5 and Equation (5),

$$
\begin{aligned}
& \bar{S}_{\alpha_{1}}=r \bar{w}_{1} s_{\alpha_{1}}=6 \times 0.195 \times s_{0.661}=s_{6 \times 0.195 \times 0.661}=s_{0.773,}, \\
& \bar{S}_{\alpha_{2}}=r \bar{w}_{2} s_{\alpha_{2}}=6 \times 0.140 \times s_{0.857}=s_{6 \times 0.140 \times 0.857}=s_{0.720}, \\
& \bar{S}_{\alpha_{3}}=r \bar{w}_{3} s_{\alpha_{3}}=6 \times 0.174 \times s_{0.655}=s_{6 \times 0.174 \times 0.655}=s_{0.684}, \\
& \bar{S}_{\alpha_{4}}=r \bar{w}_{4} s_{\alpha_{4}}=6 \times 0.175 \times s_{0.500}=s_{6 \times 0.170 \times 0.500}=s_{0.525}, \\
& \bar{S}_{\alpha_{5}}=r \bar{w}_{5} s_{\alpha_{5}}=6 \times 0.140 \times s_{0.524}=s_{6 \times 0.140 \times 0.524}=s_{0.440}, \\
& \bar{S}_{\alpha_{6}}=r \bar{w}_{6} s_{\alpha_{6}}=6 \times 0.175 \times s_{0.821}=s_{6 \times 0.175 \times 0.821}=s_{0.863}, \\
& \text { Thus, } \\
& s_{\beta_{1}}=s_{0.863}, s_{\beta_{2}}=s_{0.773}, s_{\beta_{3}}=s_{0.720}, s_{\beta_{4}}=s_{0.684}, s_{\beta_{5}}=s_{0.525}, s_{\beta_{6}}=s_{0.440},
\end{aligned}
$$

Based on the results of Steps 3 to 5, Equation (9) was used to calculate the overall value $z_{i}$ of alternative $x_{i}$ :

$$
\begin{gathered}
z_{1}=\operatorname{ILOWGA} A_{\bar{w}, w}\left(s_{\alpha_{11}}, s_{\alpha_{12}}, \ldots, s_{\alpha_{1 n}}\right)=\operatorname{ILOWGA} A_{\bar{w}, w}\left(s_{0.661}, s_{0.857}, s_{0.655}, s_{0.500}, s_{0.524}, s_{0.821}\right) \\
=\left(s_{\beta_{1}}\right)^{0} \otimes\left(s_{\beta_{2}}\right)^{0.067} \otimes\left(s_{\beta_{3}}\right)^{0.333} \otimes\left(s_{\beta_{4}}\right)^{0.333} \otimes\left(s_{\beta_{5}}\right)^{0.267} \otimes\left(s_{\beta_{6}}\right)^{0} \\
=\left(s_{0.863}\right)^{0} \otimes\left(s_{0.773}\right)^{0.067} \otimes\left(s_{0.720}\right)^{0.333} \otimes\left(s_{0.684}\right)^{0.333} \otimes\left(s_{0.525}\right)^{0.267} \otimes\left(s_{0.440}\right)^{0} \\
=s_{\left(0.863^{0} \times 0.7733^{0.067} \times 0.720^{0.333} \times 0.684^{0.333} \times 0.525^{0.267} \times 0.440^{0}\right)} \\
=s_{0.654}
\end{gathered}
$$

The other values were also obtained using the proposed method.

$z_{2}=s_{0.784}, z_{3}=s_{0.621}, z_{4}=s_{0.736}$ and $z_{5}=s_{0.855}$.

Step 7. Rank alternatives' order and provide suggestions.

The results showed that the $\mathrm{CNC}$ machine tool selection ranking order from best to worst was $A_{5}>A_{2}>A_{4}>A_{1}>A_{3}$.

\subsection{Comparisons and Discussion}

A case study verification and comparison of $\mathrm{CNC}$ machine tool selection, provided in Section 4, demonstrated the efficacy of the proposed novel group DM approach, and compared the simulation results of the proposed method (integrating induced LOWGA operator and HFLTS) with those of the LOWGA operator method and induced LOWGA operator method. Figure 2, Table 1, and Table 2 show the input data. The main differences and ranking comparison of different methods are shown in Table 6.

Table 6. The main differences and ranking comparison of the different methods.

\begin{tabular}{ccccc}
\hline & Results of Ranking the & \multicolumn{3}{c}{ Considered Factor } \\
\cline { 3 - 5 } Method Selection & $\begin{array}{c}\text { Alternatives } \\
\text { Information is } \\
\text { Hesitant }\end{array}$ & $\begin{array}{c}\text { Information is } \\
\text { Incomplete }\end{array}$ & $\begin{array}{c}\text { Consider Both the } \\
\text { Operator's Weight and } \\
\text { the Attributes' Weight } \\
\text { Synchronously }\end{array}$ \\
\hline $\begin{array}{c}\text { LOWGA operator method } \\
\text { Induced LOWGA operator } \\
\text { method }\end{array}$ & $A_{5}=A_{2}>A_{4}>A_{3}>A_{1}$ & No & No & No \\
$A_{5}>A_{2}>A_{4}>A_{3}>A_{1}$ & No & No & Yes \\
Proposed method & $A_{5}>A_{2}>A_{4}>A_{1}>A_{3}$ & Yes & Yes & Yes \\
\hline
\end{tabular}

It can be clearly seen from Tables 3-6 that the proposed new group DM method has some advantages. First, consider hesitant information. Experts provided different assessment criterion weights and criterion rating values for all alternatives, which may introduce partially hesitant information in the information aggregation procedure. Both the LOWGA operator and induced LOWGA operator methods require that the information is a single linguistic term set; moreover, these methods cannot handle hesitant information. The proposed approach applies HFLTS to handle hesitant information, and the results do not cause information distortion. 
Second, the proposed method considers incomplete information. Both the LOWGA operator and induced LOWGA operator methods only deal with complete information in the information aggregation procedure. If the assessment criterion weights and criterion rating values include incomplete information, they will be considered invalid information. However, this method may result in partially valuable information being lost, producing results that will lead to incorrect conclusions. The proposed method applies an information-supplementing process, using known information to complete incomplete information.

Finally, both consider the operator's weight and the attributes' weight synchronously. The LOWGA operator method does not consider both the operator's weight and the attributes' weight synchronously. The proposed method and induced LOWGA operator method consider both the language parameter itself and the orderly position of the language parameter. The results are thus more accurate and more reasonably reflect the actual situation.

\section{Conclusions and Further Research}

Multi-expert MCDM is a crucial issue in managerial practice, and directly influences the correctness of problem solving. In the process of information aggregation in multi-expert MCDM, information usually simultaneously contains certain, uncertain, and incomplete information provided by experts. However, traditional computing methods are unable to deal with uncertain or incomplete information, and this will cause information distortion and loss in the information aggregation process. To resolve these problems, this paper proposes a novel group DM method integrating an induced LOWGA operator and an HFLTS for solving group DM issues. In contrast to other methods, the proposed novel group DM method can simultaneously process certain, uncertain, and incomplete information in the information aggregation process. Moreover, $\mathrm{CNC}$ machine tool selection was used as an illustrative example to prove the practicality of the proposed novel group DM method. This paper also compared the calculated results with results calculated using the LOWGA operator approach and the induced LOWGA operator method. The calculation results showed that proposed novel group DM method provided a more accurate $\mathrm{CNC}$ machine tool ranking and more flexibly reflected actual world situations. To summarize, the main advantages of the proposed novel group DM method are as follows: (1) The proposed novel group DM approach can handle hesitant information provided by experts in the information aggregation process. (2) The proposed novel group DM approach can deal with incomplete information provided by experts in the information aggregation procedure. (3) The proposed method fully considers all information provided by experts, to avoid information distortion and loss. (4) The proposed method simultaneously considers the operator's weight and the attributes' weight. (5) The LOWGA operator method and induced LOWGA operator method were shown to be special cases of the proposed novel group DM approach. In future research, different practical cases will be examined to prove the practicality of the proposed novel group DM approach. Moreover, the extended concept of proposed novel group DM approach enables it to be combined with the preference relation approach for solving future group DM issues.

Author Contributions: Conceptualization, T.-C.W. and K.-H.C.; methodology, K.-H.C.; validation, T.-C.W., H.-H.L. and K.-H.C.; formal analysis, H.-H.L.; writing-original draft preparation, T.-C.W. and K.-H.C.; writing—review and editing, H.-H.L. and K.-H.C.; funding acquisition, K.-H.C. All authors have read and agreed to the published version of the manuscript.

Funding: This research was funded by Ministry of Science and Technology, Taiwan, grant number MOST 108-2410-H-145-001, MOST 109-2410-H-145-002, and The APC was funded by MOST 109-2410-H-145-002.

Conflicts of Interest: The authors declare no conflict of interest. 


\section{References}

1. Yuen, K.K.F.; Lau, H.C.W. A linguistic possibility-probability aggregation model for decision analysis with imperfect knowledge. Appl. Soft. Comput. 2009, 9, 575-589. [CrossRef]

2. Noor-E.-Alam, M.; Lipi, T.F.; Hasin, M.A.A.; Ullah, A.M.M.S. Algorithms for fuzzy multi expert multi criteria decision making (ME-MCDM). Knowl. -Based Syst. 2011, 24, 367-377. [CrossRef]

3. Halabi, A.X.; Montoya-Torres, J.R.; Obregon, N. A case study of group decision method for environmental foresight and water resources planning using a fuzzy approach. Group Decis. Negot. 2012, 21, 205-232. [CrossRef]

4. Zagonari, F.; Rossi, C. A heterogeneous multi-criteria multi-expert decision-support system for scoring combinations of flood mitigation and recovery options. Environ. Model. Softw. 2013, 49, 152-165. [CrossRef]

5. Onar, S.C.; Oztaysi, B.; Otay, I.; Kahraman, C. Multi-expert wind energy technology selection using interval-valued intuitionistic fuzzy sets. Energy 2015, 90, 274-285. [CrossRef]

6. Chang, K.H.; Chain, K.; Wen, T.C.; Yang, G.K. A novel general approach for solving a supplier selection problem. J. Test. Eval. 2016, 44, 1911-1924. [CrossRef]

7. Garg, H.; Chen, S.M. Multiattribute group decision making based on neutrality aggregation operators of q-rung orthopair fuzzy sets. Inf. Sci. 2020, 517, 427-447. [CrossRef]

8. Wang, F.; Wan, S.P. Possibility degree and divergence degree based method for interval-valued intuitionistic fuzzy multi-attribute group decision making. Expert Syst. Appl. 2020, 141, 112929. [CrossRef]

9. Morente-Molinera, J.A.; Wu, X.; Morfeq, A.; Al-Hmouz, R.; Herrera-Viedma, E. A novel multi-criteria group decision-making method for heterogeneous and dynamic contexts using multi-granular fuzzy linguistic modelling and consensus measures. Inf. Fusion 2020, 53, 240-250. [CrossRef]

10. Morente-Molinera, J.A.; Kou, G.; Perez, I.J.; Samuylov, K.; Selamat, A.; Herrera-Viedma, E. A group decision making support system for the Web: How to work in environments with a high number of participants and alternatives. Appl. Soft. Comput. 2018, 68, 191-201. [CrossRef]

11. Xiao, J.; Wang, X.L.; Zhang, H.J. Managing personalized individual semantics and consensus in linguistic distribution large-scale group decision making. Inf. Fusion 2020, 53, 20-34. [CrossRef]

12. Wen, T.C.; Chang, K.H.; Lai, H.H. Integrating the 2-tuple linguistic representation and soft set to solve supplier selection problems with incomplete information. Eng. Appl. Artif. Intell. 2020, 87, 103248. [CrossRef]

13. Zhou, L.G.; Chen, H.Y. The induced linguistic continuous ordered weighted geometric operator and its application to group decision making. Comput. Ind. Eng. 2013, 66, 222-232. [CrossRef]

14. Liu, J.P.; Chen, H.Y.; Xu, Q.; Zhou, L.G.; Tao, Z.F. Generalized ordered modular averaging operator and its application to group decision making. Fuzzy Sets Syst. 2016, 299, 1-25. [CrossRef]

15. Gitinavard, H.; Mousavi, S.M.; Vahdani, B. A new multi-criteria weighting and ranking model for group decision-making analysis based on interval-valued hesitant fuzzy sets to selection problems. Neural Comput. Appl. 2016, 27, 1593-1605. [CrossRef]

16. Ghorabaee, M.K.; Zavadskas, E.K.; Amiri, M.; Turskis, Z. Extended EDAS method for fuzzy multi-criteria decisionmaking: An application to supplier selection. Int. J. Comput. Commun. Control. 2016, 11, 358-371. [CrossRef]

17. Rodriguez, R.M.; Martinez, L.; Herrera, F. Hesitant fuzzy linguistic term sets for decision making. IEEE Trans. Fuzzy Syst. 2012, 20, 109-119. [CrossRef]

18. Torra, V. Hesitant fuzzy sets. Int. J. Intell. Syst. 2010, 25, 529-539. [CrossRef]

19. Beg, I.; Rashid, T. TOPSIS for hesitant fuzzy linguistic term sets. Int. J. Intell. Syst. 2013, 28, 1162-1171. [CrossRef]

20. Wang, J.Q.; Wang, J.; Chen, Q.H.; Zhang, H.Y.; Chen, X.H. An outranking approach for multi-criteria decision-making with hesitant fuzzy linguistic term sets. Inf. Sci. 2014, 280, 338-351. [CrossRef]

21. Montes, R.; Sanchez, A.M.; Villar, P.; Herrera, F. A web tool to support decision making in the housing market using hesitant fuzzy linguistic term sets. Appl. Soft. Comput. 2015, 35, 949-957. [CrossRef]

22. Ashtiani, M.; Azgomi, M.A. A hesitant fuzzy model of computational trust considering hesitancy, vagueness and uncertainty. Appl. Soft. Comput. 2016, 42, 18-37. [CrossRef]

23. Onar, S.C.; Buyukozkan, G.; Oztaysi, B.; Kahraman, C. A new hesitant fuzzy QFD approach: An application to computer workstation selection. Appl. Soft. Comput. 2016, 46, 1-16. [CrossRef]

24. Liu, H.B.; Rodriguez, R.M. A fuzzy envelope for hesitant fuzzy linguistic term set and its application to multicriteria decision making. Inf. Sci. 2014, 258, 220-238. [CrossRef]

25. Lee, L.W.; Chen, S.M. Fuzzy decision making and fuzzy group decision making based on likelihood-based comparison relations of hesitant fuzzy linguistic terms. J. Intell. Fuzzy Syst. 2015, 29, 1119-1137. [CrossRef] 
26. Liao, H.C.; Xu, Z.S.; Zeng, X.J.; Merigo, J.M. Qualitative decision making with correlation coefficients of hesitant fuzzy linguistic term sets. Knowl. -Based Syst. 2015, 76, 127-138. [CrossRef]

27. Chang, K.H. A more general reliability allocation method using the hesitant fuzzy linguistic term set and minimal variance OWGA weights. Appl. Soft. Comput. 2016, 56, 589-596. [CrossRef]

28. Kahraman, C.; Oztaysi, B.; Onar, S.C. A multicriteria supplier selection model using hesitant fuzzy linguistic term sets. J. Mult.-Valued Log. Soft Comput. 2016, 26, 315-333.

29. Wang, H.; Xu, Z.S. Interactive algorithms for improving incomplete linguistic preference relations based on consistency measures. Appl. Soft. Comput. 2016, 42, 66-79. [CrossRef]

30. Chang, K.H. A novel efficient approach for supplier selection problem using the OWA-based ranking technique. J. Ind. Prod. Eng. 2015, 32, 247-254. [CrossRef]

31. Yager, R.R. On ordered weighted averaging aggregation operators in multi-criteria decision making. IEEE Trans. Syst. Man Cybern. 1988, 18, 183-190. [CrossRef]

32. Xu, Z.S.; Da, W.L. The ordered weighted geometric averaging operators. Int. J. Intell. Syst. 2002, 17, 709-716. [CrossRef]

33. Cheng, C.H.; Chang, J.R. MCDM aggregation model using situational ME-OWA and ME-OWGA operators. Int. J. Uncertainty Fuzziness Knowl.-Based Syst. 2006, 14, 421-433. [CrossRef]

34. Zhou, L.G.; Chen, H.Y. Generalized ordered weighted logarithm aggregation operators and their applications to group decision making. Int. J. Intell. Syst. 2010, 25, 683-707. [CrossRef]

35. Merigo, J.M.; Gil-Lafuente, A.M.; Martorell, O. Uncertain induced aggregation operators and its application in tourism management. Expert Syst. Appl. 2012, 39, 869-880. [CrossRef]

36. Mentes, A.; Ozen, E. A hybrid risk analysis method for a yacht fuel system safety. Saf. Sci. 2015, 79, 94-104. [CrossRef]

37. Karanik, M.; Pelaez, J.I.; Bernal, R. Selective majority additive ordered weighting averaging operator. Eur. J. Oper. Res. 2016, 250, 816-826. [CrossRef]

38. Kasperski, A.; Zielinski, P. Single machine scheduling problems with uncertain parameters and the OWA criterion. J. Sched. 2016, 19, 177-190. [CrossRef]

39. Mohammed, E.A.; Naugler, C.T.; Far, B.H. Breast tumor classification using a new OWA operator. Expert Syst. Appl. 2016, 61, 302-313. [CrossRef]

40. Chang, K.H.; Cheng, C.H.; Chang, Y.C. Reliability assessment of an aircraft propulsion system using IFS and OWA tree. Eng. Optim. 2008, 40, 907-921. [CrossRef]

41. Chang, K.H. Evaluate the orderings of risk for failure problems using a more general RPN methodology. Microelectron. Reliab. 2009, 49, 1586-1596. [CrossRef]

42. Xu, Z.S. A method based on linguistic aggregation operators for group decision making with linguistic preference relations. Inf. Sci. 2004, 166, 19-30. [CrossRef]

43. Wei, C.F.; Pei, Z.; Li, H.M. An induced OWA operator in coal mine safety evaluation. J. Comput. Syst. Sci. 2012, 78, 997-1005. [CrossRef]

44. Xu, Z.S. On generalized induced linguistic aggregation operators. Int. J. Gen. Syst. 2006, 35, 17-28. [CrossRef]

45. Chang, K.H. Enhanced assessment of a supplier selection problem by integration of soft sets and hesitant fuzzy linguistic term set. Proc. Inst. Mech. Eng. Part. B-J. Eng. 2015, 229, 1635-1644. [CrossRef]

46. Farhadinia, B. Multiple criteria decision-making methods with completely unknown weights in hesitant fuzzy linguistic term setting. Knowl. -Based Syst. 2016, 93, 135-144. [CrossRef]

47. Wang, J.; Wang, J.Q.; Zhang, H.Y.; Chen, X.H. Multi-criteria decision-making based on hesitant fuzzy linguistic term sets: An outranking approach. Knowl. -Based Syst. 2015, 86, 224-236. [CrossRef]

48. Chang, S.L.; Wang, R.C.; Wang, S.Y. Applying a direct multi-granularity linguistic and strategy-oriented aggregation approach on the assessment of supply performance. Eur. J. Oper. Res. 2007, 177, 1013-1025. [CrossRef]

49. Herrera, F.; Herrera-Viedma, E.; Martinez, L. A fusion approach for managing multi-granularity linguistic term sets in decision making. Fuzzy Sets Syst. 2000, 114, 43-58. [CrossRef]

50. Wu, Z.B.; Ahmad, J.; Xu, J.P. A group decision making framework based on fuzzy VIKOR approach for machine tool selection with linguistic information. Appl. Soft. Comput. 2016, 42, 314-324. [CrossRef]

(C) 2020 by the authors. Licensee MDPI, Basel, Switzerland. This article is an open access article distributed under the terms and conditions of the Creative Commons Attribution (CC BY) license (http://creativecommons.org/licenses/by/4.0/). 